\title{
Evaluation of the Expression of the Inhibitor of Apoptosis Protein Family and Human Telomerase Reverse Transcriptase in Patients With Advanced Colorectal Adenoma
}

ARTICLE

\author{
Joon Young Choi ${ }^{1}$, Hyuk Yoon ${ }^{1}$, Gyeongjae Na ${ }^{1}$, Yoon Jin Choi ${ }^{1}$, Cheol Min Shin ${ }^{1}$, Young Soo Park ${ }^{1}$, Nayoung Kim ${ }^{1,2}$, \\ Dong Ho Lee ${ }^{1}$
}

${ }^{1}$ Department of Internal Medicine, Seoul National University Bundang Hospital, Seongnam, ${ }^{2}$ Department of Internal Medicine and Liver Research Institute, Seoul National University College of Medicine, Seoul, Korea

\begin{abstract}
Background: It has been reported that the expression of the inhibitor of apoptosis protein (IAP) family increases in patients with colon cancer. We evaluated the expression of the IAP family and human telomerase reverse transcriptase (hTERT) in normal colon mucosa from patients with advanced colorectal adenoma and investigated their features according to characteristics of advanced colorectal adenoma.

Methods: While resections of polyps were performed in patients $(n=80)$ diagnosed with advanced colorectal adenoma or carcinoma in situ, additional normal tissues were obtained from the sigmoid colon. In healthy patients $(n=16)$, blind biopsies were performed on the sigmoid colon. The expression of the IAP family, including survivin, XIAP, CIAP1, and CIAP2, and hTERT, were analyzed by real-time PCR in both groups.

Results: A total of 80 advanced colorectal adenoma patients (71.3\% male, mean age of 60.4 years) and 16 control patients were enrolled in this study. The mean ranking of CIAP2 was higher in the control group (68.88 vs. 44.43, $P=0.001)$. The expression levels of hTERT, survivin, XIAP, and cIAP from both groups showed no differences. The expression of survivin, XIAP, CIAP1, cIAP2, and hTERT depending on certain factors of advanced adenoma, including the number (two or fewer vs. three or more), size (smaller than $1 \mathrm{~cm}$ vs. larger than $1 \mathrm{~cm}$ ), grade of dysplasia (low grade adenoma vs. high grade adenoma), pathology (tubular adenoma vs. villous adenoma), and presence of endometrial intraepithelial neoplasms, showed no significant correlations in the Mann-Whitney U-test.

Conclusions: The expression of the IAP family and hTERT, except CIAP2, in the normal mucosa of patients with advanced colorectal adenoma were not different from those of the control group. There were no differences in the IAP family and hTERT according to the characteristics of advanced adenoma.
\end{abstract}

(J Cancer Prev 2017;22:98-102)

Key Words: Colonic neoplasm, Inhibitor of apoptosis proteins, Telomerase

\section{INTRODUCTION}

Colorectal cancer (CRC) is the fourth most common cause of cancer-related deaths. ${ }^{1}$ The apoptotic and anti-apoptotic signaling pathways are important in regulating carcinogenesis and determining cancer progression and prognosis. Molecules associated with apoptosis have significance as cancer diagnostic markers and therapeutic targets. The inhibitor of apoptosis protein (IAP) family includes several important molecules involved in apoptosis that might represent such targets. There is increasing evidence that the IAP family members are essential for anti-apoptotic signal transduction and that the expression of IAPs

Received April 12, 2017, Revised May 24, 2017, Accepted May 25, 2017

Correspondence to: Hyuk Yoon

Department of Internal Medicine, Seoul National University Bundang Hospital, Seoul National University College of Medicine, 82 Gumi-ro 173beon-gil, Bundang-gu, Seongnam 13620, Korea

Tel: +82-31-787-7009, Fax: +82-31-787-4051, E-mail: bodnsoul@hanmail.net, ORCID: Hyuk Yoon, http://orcid.org/0000-0002-2657-0349

Copyright (c) 2017 Korean Society of Cancer Prevention

(c) This is an Open Access article distributed under the terms of the Creative Commons Attribution Non-Commercial License (http://creativecommons.org/icenses/by-nc/4.0) which permits unrestricted non-commercial use, distribution, and reproduction in any medium, provided the original work is properly cited. 
increases, leading to poor colon cancer outcome and prognosis and to the drug resistance of tumors. ${ }^{2-4}$ X-linked IAP (XIAP), CIAP1, CIAP2, and survivin are prognostic markers of colorectal cancer, and survivin and CIAP2 are also used to predict the efficacy of anticancer treatments in CRC patients. ${ }^{5}$

CRC also undergoes a multi-step process, which is associated with genetic alterations. ${ }^{6}$ Human telomerase reverse transcriptase (hTERT) stabilizes chromosomal ends by extending the telomeric repeat sequences and plays an important role in cell survival, development and the proper functioning of various organs. ${ }^{7}$ hTERT activity is inhibited in normal somatic tissues, but hTERT expression is increased in most human tumors. ${ }^{8}$ Telomerase reactivation in the cell is associated with carcinogenesis and is an important step in the immortalization process of tumors. ${ }^{9}$

It is not known whether the expression of major IAPs or hTERT is increased in normal colon tissue of patients with advanced adenoma or carcinoma in situ. In this study, we evaluated the factors associated with apoptosis and proliferation in the normal colon mucosa of patients with advanced adenoma or carcinoma in situ compared to normal subjects and the expression levels of these factors according to the characteristics of advanced adenoma.

\section{MATERIALS AND METHODS}

\section{Study subjects}

This is a prospective study conducted at the Seoul National University Bundang Hospital, Gyeonggi-do, Korea. To be included in the study, subjects were required to meet the following criteria: (1) Patients with pathologically-proven of advanced colorectal adenoma ( 3 or more adenoma, larger than $1 \mathrm{~cm}$ adenoma, villous adenoma, high grade dysplasia) or colonic adenoma in situ. (2) Patients receiving a colonoscopy for the purpose of routine screening or irritable bowel syndrome. Patients with a history of previous $\mathrm{CRC}$, including hereditary $\mathrm{CRC}$, colon resection, and known inflammatory bowel disease (Crohn's disease or ulcerative colitis), 85 years or older or high risk of bleeding were excluded.

In the 80 patients diagnosed with advanced adenoma or carcinoma in situ, normal tissues were collected at sigmoid colon about $20 \mathrm{~cm}$ above anal verge during the polypectomy procedure. Random biopsies were also performed on an AV $20 \mathrm{~cm}$ sigmoid colon in 16 patients who underwent a colonoscopy for the purpose of medical check-up or suspected irritable bowel syndrome.
The expression levels of the major IAPs, such as XIAP, CIAP1, CIAP2, survivin and hTERT, in both groups were analyzed by real-time PCR. In addition, we analyzed whether the expression of these factors varies according to the characteristics of the advanced adenomas (number, size, grade of dysplasia, and pathology). The study protocol was approved by the Institutional Review Board of SNUH (IRB No. B-1308/215-008).

\section{Quantitative real-time PCR}

The rotor gene system (Corbett Research, Sydney, Australia) was used to run quantitative real-time polymerase chain reaction. The absolute quantification assay was chosen to analyze hTERT expression. PCR was performed using a total reaction volume of $20 \mu \mathrm{L}$ containing $1 \mathrm{x}$ TaqMan universal master mix with AmpErase uracil N-glycosylase (to prevent the re-amplification of carry-over PCR products; Applied Biosystems, Foster City, CA, USA), $600 \mathrm{nM}$ of each primer, $200 \mathrm{nM}$ TaqMan probe, and $1 \mu \mathrm{L}$ of unknown cDNA or $2 \mu \mathrm{L}$ of the standard template. All of the samples (unknown or standard) were run in duplicate and accompanied by a non-template control. Thermal cycling conditions included 2 minutes at $50^{\circ} \mathrm{C}$ and 10 minutes at $95^{\circ} \mathrm{C}$, followed by 45 cycles at $95^{\circ} \mathrm{C}$ for 15 seconds and $60^{\circ} \mathrm{C}$ for 60 minutes.

The primers for survivin were as follows: sense primer 5'-ATGGGTGCCCCGACGTTG-3'; reverse primer 5'-AGAGGCCTCAATCCATGG-3'. The XIAP primers were as follows: sense primer 5'-GGCCATCTGAGACACATGCAG-3'; reverse primer 5'-GCATTCACTAGATCTGCAACC-3'. The CIAP1 primers were as follows: sense primer 5'-GCCTGATGCTGGATAACTGG-3'; reverse primer 5'-GGCGACAGAAAAGTCAATGG-3'. The cIAP2 primers were as follows: sense primer 5'-GGACAGGAGTTCATCCGTCAA-3'; reverse primer 5'-GGGCTGTCTGATGTGGATAGC-3'. The cIAP2 primers were as follows: sense primer 5'-GCATTGGAATCAGACAGCAC-3'; reverse primer 5'-CCACGACGTAGTCCATGTTC-3'.

\section{Statistical analysis}

In each group, continuous variables such as age were compared using an ANOVA, and categorical variables, such as gender were compared using a crossover analysis (chi-squared crossover). The Mann-Whitney U-test was used to analyze the expression of IAPs and hTERT in both groups. All statistical tests were performed with the program IBM SPSS ver. 22.0 (IBM Co., Armonk, NY, USA). $P$-values of $<0.05$ was considered statistically significant.

\section{RESULTS}

Baseline characteristics of the study populations were shown 
in Table 1. There were no significant differences between the two groups with regards to sex and age $(P=0.134)$. The mean age was 60.4 years, and males comprised $71.3 \%$ of the population. Three or more adenomas were found in $46.3 \%$ of the patients, and adenomas larger than $1 \mathrm{~cm}$ were observed was $33.8 \%$ of the patients. High grade adenoma accounted for $15.0 \%$ of the cases, and villous adenoma accounted for $23.8 \%$. Early CRC was found in $11.3 \%$ of the patients.

The Mann-Whitney U-test was used to determine whether there were significant differences in the expression of hTERT,

Table 1. Baseline clinicopathologic characteristics of 80 patients with advanced colorectal adenoma

\begin{tabular}{lr}
\hline \multicolumn{1}{c}{ Characteristic } & Value \\
\hline Age $(\mathrm{yr})$ & $60.4 \pm 11.4$ \\
Sex & $57(71.3)$ \\
Male & $23(28.8)$ \\
Female & $43(53.8)$ \\
Multiple polyps & $37(46.3)$ \\
Two or less & \\
Three or more & $53(66.3)$ \\
Size & $27(33.8)$ \\
Smaller than $1 \mathrm{~cm}$ & $68(85.0)$ \\
larger than $1 \mathrm{~cm}$ & $12(15.0)$ \\
Grade of adenoma & $61(76.3)$ \\
Low grade & $19(23.8)$ \\
High grade & $9(11.3)$ \\
Pathology & $71(88.8)$ \\
Tubular adenoma & \\
Villous adenoma & $9(11.3)$ \\
IEN & $71(88.8)$ \\
IEN &
\end{tabular}

Values are presented as the mean \pm SD or number (\%). The sum of the percentages does not equal $100 \%$ because of rounding. IEN, intraepithelial neoplasia; SSA, sessile serrated adenoma.

Table 2. Expression of IAPs and hTERT in the adenoma and control groups

\begin{tabular}{lccc}
\hline Variable & $\begin{array}{c}\text { Adenoma group } \\
(\mathrm{n}=80)\end{array}$ & $\begin{array}{c}\text { Control group } \\
(\mathrm{n}=16)\end{array}$ & $P$-value \\
\hline hTERT & $39.74 \pm 122.64$ & $5.02 \pm 9.64$ & 0.159 \\
Survivin & $13.83 \pm 39.96$ & $7.82 \pm 20.05$ & 0.469 \\
XIAP & $7.80 \pm 29.43$ & $7.09 \pm 11.92$ & 0.101 \\
CIAP1 & $22.08 \pm 63.01$ & $16.90 \pm 6.72$ & 0.258 \\
cIAP2 & $1.30 \pm 3.65$ & $1.70 \pm 1.55$ & 0.001 \\
\hline
\end{tabular}

Values are presented as mean $\pm \mathrm{SD}$. IAP, inhibitor of apoptosis proteins; hTERT, human telomerase reverse transcriptase. survivin, XIAP, cIAP, or cIAP2 between the experimental group and the normal control group. There was a significant difference only in CIAP2 between the two groups, and the average ranking was rather higher in the normal control group $(P=0.001)$ (Table 2).

When assessing the expression of survivin, XIAP, CIAP1, CIAP2, and hTERT in terms of the characteristics of advanced adenoma, such as number (two or fewer vs. three or more), size (less than 1 $\mathrm{cm}$ vs. more than $1 \mathrm{~cm}$ ), grade of dysplasia (low grade adenoma vs. high grade adenoma), pathology (tubular adenoma vs. villous adenoma), and presence of endometrial intraepithelial neoplasm, we found no significant correlations using the Mann-Whitney U-test (Table 3-5).

\section{DISCUSSION}

Resistance to apoptotic stimuli is a hallmark feature of various cancers. Classical apoptosis is carried out through caspases and cysteine proteases, which induce several biochemical and molecular changes, including DNA fragmentation, ultimately leading to cell death. ${ }^{10}$ Overexpression of the IAPs is one of the mechanisms by which tumor cells acquire resistance to apoptosis. ${ }^{11}$ IAPs represent a large family of endogenous caspase

Table 3. Expression of IAPs and hTERT according to the number of polyps

\begin{tabular}{|c|c|c|c|}
\hline \multirow{2}{*}{ Variable } & \multicolumn{2}{|c|}{ No. of polyp } & \multirow{2}{*}{$P$-value } \\
\hline & Two or less & Three or more & \\
\hline hTERT & $32.05 \pm 88.03$ & $47.42 \pm 150.33$ & 0.718 \\
\hline Survivin & $14.77 \pm 44.44$ & $12.91 \pm 35.61$ & 0.737 \\
\hline XIAP & $4.91 \pm 9.85$ & $10.69 \pm 40.50$ & 0.959 \\
\hline CIAP1 & $35.86 \pm 85.48$ & $8.29 \pm 18.61$ & 0.579 \\
\hline CIAP2 & $1.35 \pm 4.52$ & $1.24 \pm 2.58$ & 0.336 \\
\hline
\end{tabular}

Values are presented as mean \pm SD. IAPs, inhibitor of apoptosis proteins; hTERT, human telomerase reverse transcriptase.

Table 4. Expression of IAPs and hTERT according to the size of polyps

\begin{tabular}{|c|c|c|c|}
\hline \multirow{2}{*}{ Variable } & \multicolumn{2}{|c|}{ Size of polyp } & \multirow{2}{*}{$P$-value } \\
\hline & Smaller than $1 \mathrm{~cm}$ & Larger than $1 \mathrm{~cm}$ & \\
\hline hTERT & $56.38 \pm 148.15$ & $7.07 \pm 13.88$ & 0.430 \\
\hline Survivin & $15.58 \pm 44.37$ & $10.59 \pm 30.64$ & 0.128 \\
\hline XIAP & $10.84 \pm 35.76$ & $1.83 \pm 4.41$ & 0.108 \\
\hline CIAP1 & $25.82 \pm 74.26$ & $14.74 \pm 30.83$ & 0.112 \\
\hline CIAP2 & $1.73 \pm 4.42$ & $0.45 \pm 0.64$ & 0.840 \\
\hline
\end{tabular}

Values are presented as mean \pm SD. IAPs, inhibitor of apoptosis proteins; hTERT, human telomerase reverse transcriptase. 
Table 5. Expression of IAPs and hTERT according to the grade of dysplasia

\begin{tabular}{|c|c|c|c|}
\hline \multirow{2}{*}{ Variable } & \multicolumn{2}{|c|}{ Grade of dysplasia } & \multirow{2}{*}{$P$-value } \\
\hline & Low grade & High grade & \\
\hline hTERT & $41.26 \pm 127.45$ & $31.13 \pm 94.87$ & 0.968 \\
\hline Survivin & $15.48 \pm 43.26$ & $4.86 \pm 6.15$ & 0.790 \\
\hline XIAP & $8.67 \pm 31.74$ & $2.86 \pm 7.40$ & 0.856 \\
\hline CIAP1 & $18.73 \pm 55.62$ & $41.04 \pm 95.96$ & 0.544 \\
\hline CIAP2 & $1.35 \pm 3.81$ & $1.02 \pm 2.66$ & 0.585 \\
\hline
\end{tabular}

Values are presented as mean \pm SD. IAPs, inhibitor of apoptosis proteins; hTERT, human telomerase reverse transcriptase.

inhibitors, including survivin, CIAP and, XIAP.

The IAP family mainly inhibits caspases 3, 7, and 9, thereby inhibiting apoptosis, whereas CIAP1 and CIAP2 inhibit caspase 8 to suppress apoptosis at the membrane receptor level. ${ }^{10}$ In particular, cIAP2 is widely expressed in normal tissues and has a role as a prognostic factor in CRC as well as in evaluating the response to chemotherapy. ${ }^{12}$

While the physiological role of IAPs in normal tissues is unclear, their role in cancerous tissues appears to be different given that they are expressed rapidly and extensively in external tissues with high apoptotic rates. ${ }^{13}$

XIAP might play important roles in tumor progression and could be a potential target for the treatment of human cancers. Therapeutic strategies that interrupt XIAP expression or function have been examined as an adjuvant to conventional chemotherapy and radiation-based cancer therapy.

In addition, survivin is a risk factor for future $\mathrm{CRC}$ in colorectal adenoma patients. The biological impact of survivin on CRC risk is consistent with the ability of neoplastic cells to avoid apoptosis and achieve limitless replicative potential. ${ }^{14}$

In most tumor cells, telomeres are short and stable, and telomere length is an important indicator of the telomerase activity and is useful for the prognosis of malignant cases, including colorectal neoplasia. ${ }^{15}$ hTERT acts as a reverse transcriptase in the elongation of telomeres, which prevents their loss during replication and prevents the end-to-end fusion, recombination, and degradation of chromosomes. In CRC development, hTERT activation occurs during the progression from low- to high-grade IEN in adenomas and steadily increases with progression of dysplasia and colorectal carcinogenesis invasion. ${ }^{16}$

In the case of hTERT, one of the main evaluation parameters of this study, mRNA expression is increased by approximately 1.48 times when comparing the surrounding normal mucosa tissue to the expression found in CRC and adenoma tissue. ${ }^{17}$
It is not known whether the expression of major IAPs or hTERT is increased in the normal colon tissues of adenoma patients. We analyzed the expression levels of major IAPs, such as XIAP, cIAP1, CIAP2, survivin, and hTERT in both groups by real-time PCR to investigate the factors affecting colon cancer development. In the Mann-Whitney U-test, the cIAP2 levels in the control group and the experimental group were significantly different, but the average ranking was higher in the control group. In this regard, it has been reported that the expression of CIAP2 in RCC tissue is decreased relative to normal tissue by $17.0 \%{ }^{18}$ The association of the IAP family and hTERT with carcinogenesis may seem obvious, but we need to study the inverse relationship, as described above.

In conclusion, the expression of major IAPs controlling apoptosis and hTERT, which regulates cell proliferation, on normal colonic mucosa tissues of patients with advanced colorectal adenoma or carcinoma in situ is not different from the control group. The increased expression of these proteins previously reported in cancer patients appears to be initiated at a relatively late stage in the adenoma-carcinoma sequence of colon cancer. There are no differences in the IAP family and hTERT when taking into account the characteristics of advanced adenoma. This study helps to understand the carcinogenic process of colon cancer and identify risk factors for CRC in advanced adenocarcinoma patients.

\section{ACKNOWLEDGMENTS}

This study was supported by the SNUBH Research Fund (No. 02-2013-090) and the National Research Foundation of Korea grant for the Global Core Research Center funded by the Korean government (MSIP) (No. 2011-0030001).

\section{CONFLICTS OF INTEREST}

No potential conflicts of interest were disclosed.

\section{REFERENCES}

1. Torre LA, Bray F, Siegel RL, Ferlay J, Lortet-Tieulent J, Jemal A. Global cancer statistics, 2012. CA Cancer J Clin 2015:65:87-108.

2. Karasawa H, Miura K, Fujibuchi W, Ishida K, Kaneko N, Kinouchi $\mathrm{M}$, et al. Down-regulation of cIAP2 enhances 5-FU sensitivity through the apoptotic pathway in human colon cancer cells. Cancer Sci 2009;100:903-13.

3. Varfolomeev E, Blankenship JW, Wayson SM, Fedorova AV, Kayagaki N, Garg P, et al. IAP antagonists induce autoubiquitination of c-IAPs, NF-kappaB activation, and TNFalpha-dependent apoptosis. Cell 2007;131:669-81. 
4. Oi G, Tuncel H, Aoki E, Tanaka S, Oka S, Kaneko I, et al. Intracellular localization of survivin determines biological behavior in colorectal cancer. Oncol Rep 2009;22:557-62.

5. Hunter AM, LaCasse EC, Korneluk RG. The inhibitors of apoptosis (IAPs) as cancer targets. Apoptosis 2007;12:1543-68

6. Vogt PK. Cancer genes. West J Med 1993;158:273-8.

7. Greider CW, Blackburn EH. Telomeres, telomerase and cancer. Sci Am 1996;274:92-7.

8. Kim NW, Piatyszek MA, Prowse KR, Harley CB, West MD, Ho PL, et al. Specific association of human telomerase activity with immortal cells and cancer. Science 1994:266:2011-5.

9. Shay JW, Wright WE. The reactivation of telomerase activity in cancer progression. Trends Genet 1996;12:129-31

10. Smolewski $\mathrm{P}$, Robak $\mathrm{T}$. Inhibitors of apoptosis proteins (IAPs) as potential molecular targets for therapy of hematological malignancies. Curr Mol Med 2011;11:633-49.

11. Xiang G, Wen X, Wang H, Chen K, Liu H. Expression of X-linked inhibitor of apoptosis protein in human colorectal cancer and its correlation with prognosis. J Surg Oncol 2009;100:708-12.

12. Miura K, Fujibuchi $\mathrm{W}$, Ishida $\mathrm{K}$, Naitoh $\mathrm{T}$, Ogawa $\mathrm{H}$, Ando T, et al. Inhibitor of apoptosis protein family as diagnostic markers and therapeutic targets of colorectal cancer. Surg Today 2011:41:175-82.

13. Vischioni B, van der Valk P, Span SW, Kruyt FA, Rodriguez JA, Giaccone G. Expression and localization of inhibitor of apoptosis proteins in normal human tissues. Hum Pathol 2006;37:78-86.

14. Altieri DC. The case for survivin as a regulator of microtubule dynamics and cell-death decisions. Curr Opin Cell Biol 2006;18: 609-15.

15. Boldrini L, Faviana P, Gisfredi S, Zucconi Y, Di Quirico D, Donati $\mathrm{V}$, et al. Evaluation of telomerase mRNA (hTERT) in colon cancer. Int J Oncol 2002;21:493-7.

16. Kanamaru T, Tanaka K, Kotani J, Ueno K, Yamamoto M, Idei Y, et al. Telomerase activity and hTERT mRNA in development and progression of adenoma to colorectal cancer. Int J Mol Med 2002; 10:205-10

17. Saleh S, Lam AK, Ho YH. Real-time PCR quantification of human telomerase reverse transcriptase (hTERT) in colorectal cancer. Pathology 2008;40:25-30.

18. Kempkensteffen C, Hinz S, Christoph F, Köllermann J, Krause H, Schrader M, et al. Expression parameters of the inhibitors of apoptosis CIAP1 and cIAP2 in renal cell carcinomas and their prognostic relevance. Int J Cancer 2007;120:1081-6. 